\title{
Sentido de pertenencia escolar entre niños, niñas y adolescentes en Chile: perfiles e itinerarios mediante árboles de clasificación
}

Sense of Belonging at School Among Children and Adolescents in Chile: Profiles and Paths Through Decision Tree

O Ambiente de Sala de Aula em Escolas Públicas: Voltada a um Ensino mais Refletivo

Carlos Rodríguez-Garcés* iD orcid.org/0000-0002-9346-0780 Denisse Espinosa-Valenzuela** iD orcid.org/0000-0003-1486-7046

Geraldo Padilla-Fuentes*** (iD orcid.org/0000-0003-0882-1818

Para citar este artículo: Rodríguez-Garcés, C., Espinosa-Valenzuela, D. y Padilla-Fuentes, G. (2O21). Sen tido de pertenencia escolar entre niños, niñas y adolescentes en Chile: perfiles e itinerarios mediante árboles declasificación. RevistaColombianadeEducación, /(81),103-122.https://doi.org/10.17227/rce.num81-10256

Recibido: 03/07/2019

(c) $(1) \Theta$

* Doctor en Educación. Director del Centro de Investigación Educativa CIDCIE, Universidad del Bío-Bío, Chile. Correo: carlosro@ubiobio.cl

** Profesora en Educación Media en Castellano y Comunicación. Investigadora Adscrita al Centro de Investigación CIDCIE, Universidad del Bío-Bío, Chile. Correo: daespinosa@ubiobio.cl

*** Trabajador social. Investigador Adscrito al Centro de Investigación CIDCIE, Universidad del Bío-Bío, Chile. Correo: gpadilla@ubiobio.cl 


\begin{abstract}
Resumen
Con el objetivo de indagar sobre el sentido de pertenencia que manifiestan hacia su escuela niños, niñas y adolescentes de Chile de séptimo grado básico a tercer grado medio, este artículo modela, desde una perspectiva cuantitativa, los itinerarios y perfiles que estructuran el apego a la unidad educativa mediante la técnica de árboles de clasificación. El sentido de pertenencia hacia la escuela es un fenómeno multifactorial y sustancial para el desarrollo íntegro de los estudiantes, el cual incide no solo sobre aspectos académicos, sino también sobre los niveles de bienestar subjetivo y vínculos entre la comunidad. Los resultados informan que no todos los estudiantes forjan un vínculo afectivo estable y duradero con su escuela. No obstante, en la medida en que mejoran sus niveles de satisfacción escolar, apoyo docente percibido y evaluación de calidad educativa, este sentido de pertenencia e identidad crece. Las conclusiones destacan cómo ambos fenómenos tienen un impacto positivo en la vida escolar, tanto por la mayor implicación que estimulan entre los actores, como por la satisfacción personal que producen en niños, niñas y adolescentes durante la etapa escolar.
\end{abstract}

\section{Palabras clave}

educación de calidad; relaciones en la comunidad escolar; bienestar estudiantil; sentido de pertenencia; ambiente de clases

\section{Keywords}

quality education; schoolcommunity relationship; student well-being; sense of community; classroom environment

\begin{abstract}
In order to inquire into the sense of belonging of Chilean children and adolescents from seventh year of primary school to third year of secondary school towards their school, this article models, from a quantitative perspective, the characteristics and profiles that build up the attachment to the educational unit through the classification tree method. The sense of belonging to the school is a multifactorial and substantial phenomenon for the comprehensive development of students. Its impact is not limited to academic aspects, but it also involves the levels of subjective well-being and the bonds within the community. According to the results, not all of the students forge a stable and lasting affective bond with their school. However, as their levels of school satisfaction, perceived teacher support, and evaluation of educational quality improve, the sense of belonging and identity grows. The conclusions highlight that both phenomena have a positive impact on school life because of the greater involvement they stimulate among the actors, as well as for the personal satisfaction they produce in children and adolescents during the school stage.
\end{abstract}

\section{Resumo}

Com o objetivo de investigar o sentido de pertencimento que meninos, meninas e adolescentes chilenos de sétima a terceira séries demonstram em relação à escola, este artigo modela, desde uma perspectiva quantitativa, os itinerários e perfis que estruturam o apego ao unidade educacional usando a técnica da árvore de classificação. O sentimento de pertencimento à escola é um fenômeno multifatorial e substancial para o desenvolvimento integral dos alunos que afeta não só os aspectos acadêmicos, mas também os níveis de bem-estar subjetivo e os vínculos entre a comunidade. Os resultados mostram que nem todos os alunos estabelecem um vínculo estável e duradouro com a escola. No entanto, à medida que melhoram os níveis de satisfação escolar, o apoio percebido dos professores e a avaliação da qualidade educacional, esse sentimento de pertencimento e identidade aumenta. As conclusões evidenciam como ambos fenômenos têm impacto positivo na vida escolar, tanto pelo maior envolvimento que estimulam entre os atores, quanto pela satisfação pessoal que produzem em meninos, meninas e N. ${ }^{\circ} 81$ adolescentes durante a fase escolar.

\section{Palavras-chave}

educação de qualidade; relacionamento na comunidade escolar; bem-estar do aluno; sentimento de pertencimento; ambiente de sala de aula 


\section{Introducción}

La educación es uno de los pilares clave para el desarrollo cultural, económico y político de los territorios. Ejerce tal influencia sobre los proyectos de desarrollo, que históricamente ha existido una preocupación sociopolítica por mejorar los sistemas encargados de distribuirla, evaluarla y optimizarla (Tedesco, 2012). Ya sea desde una perspectiva institucional, práctica o social, la educación acuña la responsabilidad de proveer las herramientas y capacidades necesarias para que todos alcancen un desarrollo integral y acorde a los derechos, obligaciones y necesidades que definen el ser-ciudadano. Por ello, los nuevos enfoques educativos buscan construir una perspectiva multidimensional y compleja que sustente las prácticas de intervención en la institución escolar, especialmente en lo referido al currículo, la cultura y el ethos escolar y las redes (Keating et al., 2009).

Las experiencias de aprendizaje y socialización que tienen lugar en la etapa escolar delimitan parte importante de las actitudes y comportamientos con que se enfrenta la vida cívica en la juventud y en la adultez (Keating y Janmaat, 2016), y aportan a la convivencia, la colaboración y el cumplimiento de desafíos en los diferentes contextos de una sociedad de conocimiento (PISA, 2015). Estas contribuciones son sustanciales para el trabajo que oficinas gubernamentales, organizaciones civiles y actores locales realizan con la finalidad de mejorar la integración y el desarrollo personal y social de los estudiantes, así como la calidad de los procesos educativos. Esto implica diseñar o alinear políticas y programas que centralicen la dimensión subjetiva y relacional que tiene la escuela como lugar de convivencia (Bellei, 2013; Carrasco y González, 2017; Espinoza, 2015; Murillo y Duk, 2016).

En este contexto, el aprendizaje y los contenidos curriculares han sido objetos de revisión e intervención a fin de facilitar su significación en los alumnos, destacando la escuela como matriz de identidades y comunidad que se preocupa por la autoestima, la motivación y la pertenencia que sienten sus miembros (Valenzuela, 2007). Este marco de comprensión y trabajo acerca de la educación y sus deberes, para el caso de Chile, se estableció como estatuto ministerial a través de varias iniciativas, entre las que destaca la Ley general de educación en el 2009 y la creación de la Agencia de Calidad de la Educación en el 2013. Estos cuerpos legislativos e institucionales cimentaron el concepto de mirada amplia de calidad en estrecha relación con la percepción y valoración que los actores, especialmente estudiantes, hacen de su comunidad escolar y el vínculo que mantienen con ella. 


\section{Evidencias del vínculo entre escuela y sentido de pertenencia}

El contexto educativo descrito sirve de base para la instalación de nuevas líneas de investigación e intervención en el campo escolar, esfuerzos orientados a mejorar, por un lado, las condiciones de educabilidad y desarrollo cognitivo de los estudiantes y, por otro, aspectos del desarrollo personal y social que se propicia en los colegios.

Aquí es donde aparecen los trabajos vinculados al sentido de pertenencia a la escuela como factor cardinal para el desarrollo de las comunidades educativas. Autores como Spady (1971), Tinto (1975) y Finn (1989) han aportado evidencias para considerar que a mayores niveles de involucramiento, compromiso y filiación del estudiante hacia su escuela se registran mejores índices de crecimiento y progreso educativo.

En la última década ha crecido el interés por medir el sentido de pertenencia, sobre todo por su influencia en el bienestar subjetivo de los estudiantes. Así, la calidad del vínculo entre estudiante y escuela se ha vuelto parte sustancial de los enfoques orientados a evaluar el desarrollo personal y social en etapas escolares, entendiendo por sentido de pertenencia la relación de identificación que los miembros de la comunidad educativa, especialmente estudiantes, tienen con la institución a la que asisten (Agencia de Calidad de la Educación, 2017). A este sentimiento de afiliación se atribuyen características positivas para la trayectoria escolar, satisfacción con la vida, confianza y predisposición hacia el aprendizaje, por lo que activamente se ha incorporado en las políticas de gestión y medición educativa (Cordano, 2017).

A nivel internacional, la evidencia disponible informa que el vínculo afectivo e identitario que los estudiantes forjan con la escuela tiene un impacto positivo sobre el aprendizaje, la convivencia y los climas áulicos (Blanco, J. 2010; Hopenhayn y Sojo, 2011; López de Mesa et al., 2013; Orellana y Segovia, 2014). Con el fortalecimiento de estos vínculos se incrementan la satisfacción personal y el compromiso colectivo y académico, y disminuyen el abandono, las experiencias de fracaso escolar, la inseguridad personal y los problemas en el clima de convivencia (Aron et al., 2012; Bailey et al., 2015; Carrasco y Luzón, 2019; Fernández et al., 2010; John et al., 2018; Villalobos et al., 2016).

Otros hallazgos exponen que el sentido de pertenencia es sustancial para evitar sentimientos de incomodidad e insatisfacción con la escuela (Johnson, 2010), nutrir la noción de que se pertenece a un lugar donde se es aceptado y valorado (Quaresma, 2014; Riley, 2013), y colaborar con la obtención de aprendizajes más significativos (Sánchez et al., 2005). 
Recientes investigaciones sobre sentido de pertenencia en Chile refuerzan el principio de que a mayor filiación o arraigo con el lugar donde se aprende y convive, mejores resultados académicos, relacionales y subjetivos, principalmente a raíz de mediciones de satisfacción entre estudiantes, docentes y apoderados (López et al., 2014). No obstante, este sentimiento identitario y de reconocimiento entre actores se encuentra obstaculizado por la alta segregación y segmentación del sistema escolar, sesgos de selectividad y competencia entre establecimientos (Assaél, 2011; Bellei, 2015; Valenzuela et al., 2010). Así, en lugar de construir comunidades inclusivas, el sentido de pertenencia estaría condicionado por el grupo al que se pertenece, los cuales se excluyen y rechazan mutuamente (Peña, 2007).

Con base en estas evidencias, tanto nacionales como internacionales, es que el sentido de pertenencia se considera un fenómeno de interés entre organismos y actores vinculados a las comunidades educativas, sobre todo pensando en cómo construir espacios de convivencia, interacción y corresponsabilidad nutritivos para el desarrollo personal y social de los estudiantes en paralelo a la preocupación clásica por optimizar aspectos pedagógicos y de calidad educativa (Carbajal, 2010; Fierro, 2013).

En síntesis, teniendo en cuenta el contexto educativo nacional y la importancia del sentido de pertenencia, este artículo busca conocer cuál es el nivel de conformidad, o sentido de pertenencia, que niños, niñas y adolescentes (en adelante NNA) tienen hacia la institución educativa a la que asisten. Los resultados provienen de la encuesta sobre Bienestar Subjetivo que el Programa de Naciones Unidades (PNUD) en Chile aplicó a NNA de diferentes colegios y el uso de un diseño metodológico cuantitativo mediante la técnica de análisis multivariante árboles de decisión. Se buscó responder a preguntas acerca del efecto que tendrían aspectos institucionales y perceptivos de los estudiantes sobre el sentimiento de afiliación a la escuela, cómo se relaciona el bajo sentido de pertenencia con el deseo de permanecer o no en la escuela, qué características definen el fenómeno de interés y permiten hacer diferencias significativas para atender distintos grupos en los establecimientos.

\section{Metodología}

\section{Instrumento}

Enmarcado en la Política Nacional de Niñez y Adolescencia 2015-2025 del Gobierno de Chile, se construyó esta investigación a partir de los resultados de la Primera Encuesta de Desarrollo Humano en Niños, Niñas y Adolescentes (EDH-NNA) que fue aplicada por el Programa de Naciones Unidas para el Desarrollo (PNUD) y por el Consejo Nacional de la Infancia 
en 2017 a NNA entre los 12 y los 17 años, la cual tuvo por objetivo evaluar desde la propia experiencia de los NNA, diferentes aspectos relativos a su desarrollo humano en los contextos personal, familiar y escolar. Los datos se obtienen a través de entrevistas cara a cara asistidas por dispositivos electrónicos (CAPI).

\section{Muestra}

La encuesta fue aplicada a un total de 3073 NNA pertenecientes a 272 establecimientos educativos (municipales, particulares subvencionados y particulares pagados de todas las regiones del país). La muestra se obtuvo mediante procesos trietápicos y probabilísticos, aplicada a estudiantes de séptimo grado básico a tercer grado medio. La encuesta reporta un margen de error de $\pm 1,8$ puntos porcentuales, un nivel de confianza del $95 \%$, una tasa de respuesta del 73,0 \% y de rechazo del $4 \%$.

Tabla 1.

Caracterización de la muestra (\%)

\begin{tabular}{|c|c|c|c|}
\hline \multirow[t]{2}{*}{ Zona } & \multicolumn{3}{|c|}{ Sexo } \\
\hline & 88,8 & Hombre & 52,9 \\
\hline Rural & 11,2 & Mujer & 47,1 \\
\hline Rama educativa & \multicolumn{3}{|c|}{ Nivel educativo } \\
\hline Humanista & 92,4 & Básico & 66,4 \\
\hline Técnico profesional & 7,6 & Secundario & 33,6 \\
\hline \multicolumn{4}{|l|}{ Dependencia } \\
\hline Municipal & 43,8 & & \\
\hline particular subvencionado & 50,9 & & \\
\hline Particular pagado & 5,2 & & \\
\hline
\end{tabular}

Fuente: elaboración propia con base en resultados de la EDH-NNA (2017).

Mayoritariamente, la muestra la componen estudiantes de zonas urbanas $(88,8 \%)$, de la rama científico-humanista (92,4\%); un tercio cursan estudios secundarios $(33,6 \%$ ) con leve predominancia del sexo masculino $(52,9 \%)$ y de colegios particulares $(56,1 \%)$.

\section{Procedimiento}

El análisis de los datos de la encuesta EDH-NNA se inició con la indagación descriptiva de los ítems que componen la variable dependendiente (Sentido de pertenencia), buscando establecer diferencias estadísticamente significativas con otras variables de estudio. 
Posteriormente, mediante la técnica estadística multivariante árboles de clasificación, se diagramó una estructura jerárquica según factores con alta capacidad de discriminación y predicción de la variable modelada. Esta representación de resumen para grandes volúmnes de datos maximiza la distancia entre grupos formando nodos, ramas y hojas capaces de estructurar itinerarios y definir perfiles (Gervilla y Palmer, 2009; Han y Kamber, 2006). Estimamos que esta información sería de utilidad para el diseño e implementación de propuestas de intervención educativa tanto de profesores como de profesionales asistentes de la educación, para actuar sobre el sentido de pertenencia mejorando los niveles de apego, implicación y vínculo emocional que los estudiantes tienen y manifiestan con respecto a su unidad educativa.

El método de segmentación jerárquica utilizado para el desarrollo del árbol fue Chi-square Automatic Interaction Detection (Chaid), basado en chi cuadrado para seleccionar a los predictores. La variable criterio es el Sentido de pertenencia que reportan NNA. Esta se dicotomizó para fines analíticos y se estableció como punto de corte 0,70; se tipificaron las categorías como Permanece o Abandona ,según se posicione el índice por debajo o por encima del límite establecido.

Tanto la variable modelada como sus predictores fueron sometidos a contraste de asimetría y verificación del cumplimiento de la regla 90/10 dada su naturaleza categorial. Con el propósito de evitar el sobreajuste del modelo, se establecieron reglas de 3 niveles con podas cuando no se superaran los 200 casos para el nodo padre y 100 para el nodo hijo. Además, se trabajó solo con aquellos casos que reportan información completa para el conjunto de variables insertadas en el modelo. Esta supresión de valores perdidos dio como resultado una muestra de 2.588 NNA.

El modelo resultante terminó por seleccionar 5 predictores de un total de 10 iniciales, conformando un árbol con 10 nodos y 6 de ellos terminales. El nodo más pequeño agrupa el 4,6 \% de los casos. El modelo tiene adecuada capacidad predictiva, clasificando correctamente al $75,9 \%$ de los casos, y es particularmente alto en su nivel de sensibilidad $(94,6 \%)$.

\section{Variables}

Dada la naturaleza multivariante y exploratoria del modelamiento de árboles de clasificación, se incluyeron como predictores un conjunto de variables que la literatura reporta como teóricamente relevantes (véase la tabla 2). 
Tabla 2.

\begin{tabular}{|c|c|c|}
\hline Variable & Operacionalización (categorías que comprende) & $\begin{array}{l}\text { Significancia } \\
\text { Posición jerárquica } \\
\text { [Simetrías] }\end{array}$ \\
\hline $\begin{array}{l}\text { Sentido de } \\
\text { pertencia } \\
\text { escolar }\end{array}$ & $\begin{array}{l}\text { Nivel de identificación y apego que el estudiante } \\
\text { declara tener respecto de su institución educativa } \\
\text { (escuela-liceo). Manifestación dicotómica según } \\
\text { exprese Permanecer (O) o Abandonar (1). }\end{array}$ & $\begin{array}{l}\text { Variable dependiente } \\
{[\text { Permanecer }=72,7 \% \text {; }} \\
\text { Abandonar }=27.3 \%]\end{array}$ \\
\hline $\begin{array}{l}\text { Calidad } \\
\text { educativa }\end{array}$ & $\begin{array}{l}\text { Valoración que hacen los nNA sobre la experiencia, } \\
\text { los recursos y las oportunidades que brinda la } \\
\text { institución educativa. Índice continuo estandarizado } \\
\text { que se dicotomiza en Alta y Baja, estableciendo la } \\
\text { puntuación 0,70 como valor de corte. }\end{array}$ & $\begin{array}{l}\text { Significativa } \\
\text { Tercer nivel } \\
\text { [Baja }=24,7 \% \text {; } \\
\text { alta }=75,3 \%]\end{array}$ \\
\hline $\begin{array}{l}\text { Apoyo familiar } \\
\text { percibido }\end{array}$ & $\begin{array}{l}\text { Índice continuo estandarizado construido por } \\
12 \text { ítems tipo Likert de respuesta graduada en } \\
5 \text { niveles. En el modelo Ac es dicotomizada, } \\
\text { estableciéndose como punto de corte 0,70. }\end{array}$ & $\begin{array}{l}\text { No significativa } \\
\text { [Bajo }=17,5 \% \\
\text { alto }=82,5 \%]\end{array}$ \\
\hline $\begin{array}{l}\text { Apoyo } \\
\text { docente } \\
\text { percibido }\end{array}$ & $\begin{array}{l}\text { Índice continuo estandarizado construido por } 7 \\
\text { ítems tipo Likert con } 5 \text { niveles de respuesta. Es } \\
\text { dicotomizada con punto de corte 0,70. }\end{array}$ & $\begin{array}{l}\text { Significativa } \\
\text { Segundo nivel } \\
\text { [Bajo = } 18,8 \% \text {; } \\
\text { alto }=81,2 \% \text { ] }\end{array}$ \\
\hline $\begin{array}{l}\text { Satisfacción } \\
\text { con los amigos }\end{array}$ & $\begin{array}{l}\text { Variable escalar que transita entre } 1 \text { y } 10 \text { para } \\
\text { evaluar la satisfacción hacia las relaciones } \\
\text { de amistad. En el modelo es dicotomizada, } \\
\text { estableciéndose como punto de corte } 7 .\end{array}$ & $\begin{array}{l}\text { No significativa } \\
\text { [Baja }=16,9 \% ; \\
\text { alta }=83,1 \%]\end{array}$ \\
\hline $\begin{array}{l}\text { Satisfacción } \\
\text { escolar }\end{array}$ & $\begin{array}{l}\text { Variable escalar que transita entre } 1 \text { y } 10 \text { para } \\
\text { evaluar la satisfacción hacia la experiencia escolar. } \\
\text { Es dicotomizada con punto de corte } 7 .\end{array}$ & $\begin{array}{l}\text { Significativa } \\
\text { Primer nivel } \\
\text { [Baja }=18,1 \% \text {; } \\
\text { alta }=81,9 \%]\end{array}$ \\
\hline $\begin{array}{l}\text { Satisfacción } \\
\text { con la vida }\end{array}$ & $\begin{array}{l}\text { Variable escalar que transita entre } 1 \text { y } 10 \text { para } \\
\text { evaluar la satisfacción con la vida. Es dicotomizada } \\
\text { con punto de corte } 7 .\end{array}$ & $\begin{array}{l}\text { No significativa } \\
\text { [Baja }=17,7 ; \\
\text { alta }=82,3 \%]\end{array}$ \\
\hline Autoestima & $\begin{array}{l}\text { Índice continuo estandarizado construido por } \\
5 \text { ítems tipo Likert de respuesta graduada en } \\
5 \text { niveles. En el modelo Ac es dicotomizada, } \\
\text { estableciéndose como punto de corte O,70, } \\
\text { distinguiendo baja y alta autoestima. }\end{array}$ & $\begin{array}{l}\text { No significativa } \\
{[\mathrm{Baja}=39,3 \% \text {; alta }=} \\
60,7 \%]\end{array}$ \\
\hline $\begin{array}{l}\text { Nivel } \\
\text { educativo }\end{array}$ & $\begin{array}{l}\text { Variable de segmentación que da cuenta del ciclo } \\
\text { escolar en que se encuentra el estudiante: Primario } \\
\left(7 .^{\circ} \text { y } 8 .^{\circ} \text { básico) y Secundario }\left(1 .^{\circ}, 2 .^{\circ} \text { y } 3 .^{\circ} \text { de }\right.\right. \\
\text { Enseñanza Media). }\end{array}$ & $\begin{array}{l}\text { Significativa } \\
\text { Segundo nivel } \\
\text { [Básico = 65,2\%; } \\
\text { medio = 34,8\%] }\end{array}$ \\
\hline Género & Variable basal de caracterización & $\begin{array}{l}\text { Significativa } \\
\text { Tercer nivel } \\
{[\text { Hombre }=52,7 \% \text {; }} \\
\text { Mujer }=47,3 \%]\end{array}$ \\
\hline Dependencia & $\begin{array}{l}\text { Tipología de administración escolar según } \\
\text { naturaleza del financiamiento y provisión del } \\
\text { servicio educativo. }\end{array}$ & $\begin{array}{l}\text { No significativa } \\
\text { [Municipal = 42,8\%; } \\
\text { part. subvencio- } \\
\text { nado = 51,5\%; part. } \\
\text { pagado = 5,7\%] }\end{array}$ \\
\hline
\end{tabular}




\section{Análisis y discusión de resultados}

Una grata experiencia escolar puede verse afectada por distintos factores, siendo el sentido de pertenencia que manifiestan NNA hacia su institución educativa el fenómeno de interés para este artículo. Este factor, entendido como el sentirse parte de la escuela (belonging) e integrar sus valores y normas (valuing) como miembro del grupo (Quaresma y Zamorano, 2016), busca posicionarse como parte sustancial de la mirada amplia que la Ley general de educación y los Indicadores de Desarrollo Personal y Social (IDPS) han promovido en la medición de calidad educativa en Chile.

Al respecto, tal y como informa la EDH-NNA del PNUD en Chile, 1 de cada 4 estudiantes $(27,3 \%)$ reporta que le gustaría cambiarse de escuela. Si bien esta manifestación encuentra matices dependiendo de los contextos social, cultural y pedagógico de cada estudiante, sería expresión de la débil capacidad atribuida a la escuela para establecer y nutrir relaciones más o menos cercanas (Kia-Keating y Ellis, 2007).

Al ser la escuela una institución formal donde el individuo establece contacto primario con otros significativos ajenos al grupo familiar y adquiere experiencias basales para su desarrollo integral e inserción social (Gilbert, 2010), resulta preocupante la alta prevalencia de estudiantes que desearían abandonar la institución educativa de la cual son parte. Algunos factores que pueden estar influenciando este tipo de sentimiento entre NNA son la exposición a ambientes de convivencia deteriorada, climas áulicos hostiles y/o recurrentes episodios de violencia (Román y Murillo, 2011; Tijmes, 2012), eventos que resquebrajan la base de seguridad personal y colectiva. Así también, dificultan el establecimiento de lazos afectivos cercanos entre profesores y compañeros, inhiben actitudes positivas hacia el aprendizaje y compromiso escolar (Luckner y Pianta, 2011).

El sentido de pertenencia hacia la escuela, además de ser expresión del nivel de adhesión al proyecto educativo institucional es un fenómeno polisémico y polimorfo que encuentra sus particularidades en cada contexto (Juvonen, 2006). Por esta razón, la revisión de aquellos elementos que lo influencian y condicionan debe incluir además de características individuales, atributos colectivos e institucionales del lugar donde se desarrolla la experiencia educativa. Por ello, y a fin de determinar los pesos discriminatorios que distintos factores mencionados por la teoría tienen sobre el sentido de pertenencia, esta investigación aplica un modelo multivariante de árboles de clasificación. De este modo se identifican los perfiles de NNA que expresan mayor y menor sentido de pertenencia hacia su escuela, con énfasis en los deseos de migrar que traería consigo el bajo sentido de afiliación. 
En primera instancia, encontramos que el modelo clasificatorio de árboles de segmentación (véase la gráfica 1) vincula el sentido de pertenencia con variables como Satisfacción escolar, Nivel educativo, Apoyo docente percibido, Género y apreciación de la calidad educativa del establecimiento. Por su parte, atributos asociados al Apoyo familiar percibido, la Satisfacción con los amigos, la Autoestima y el Tipo de establecimiento no reportaron la significancia esperada. Esta situación se explica en parte por el principio de organización jerárquica de la técnica y sus condiciones de poda para evitar sobreajustes.

Así, la Satisfacción escolar se posiciona como la variable de mayor capacidad discriminativa $\left[x^{2}=237,780 ; p=0,000\right]$, de modo tal que aqueIlos estudiantes que manifiestan un bajo nivel de conformidad o bienestar subjetivo con su institución educativa registran el mayor riesgo de migrar $(56,1 \%)$. Esta situación contrasta significativamente cuando el alumnado otorga a su establecimiento una alta puntuación en esta variable, en este caso el deseo de cambiarse alcanza tan solo un 21,0 \% de adhesión (nodo 2).

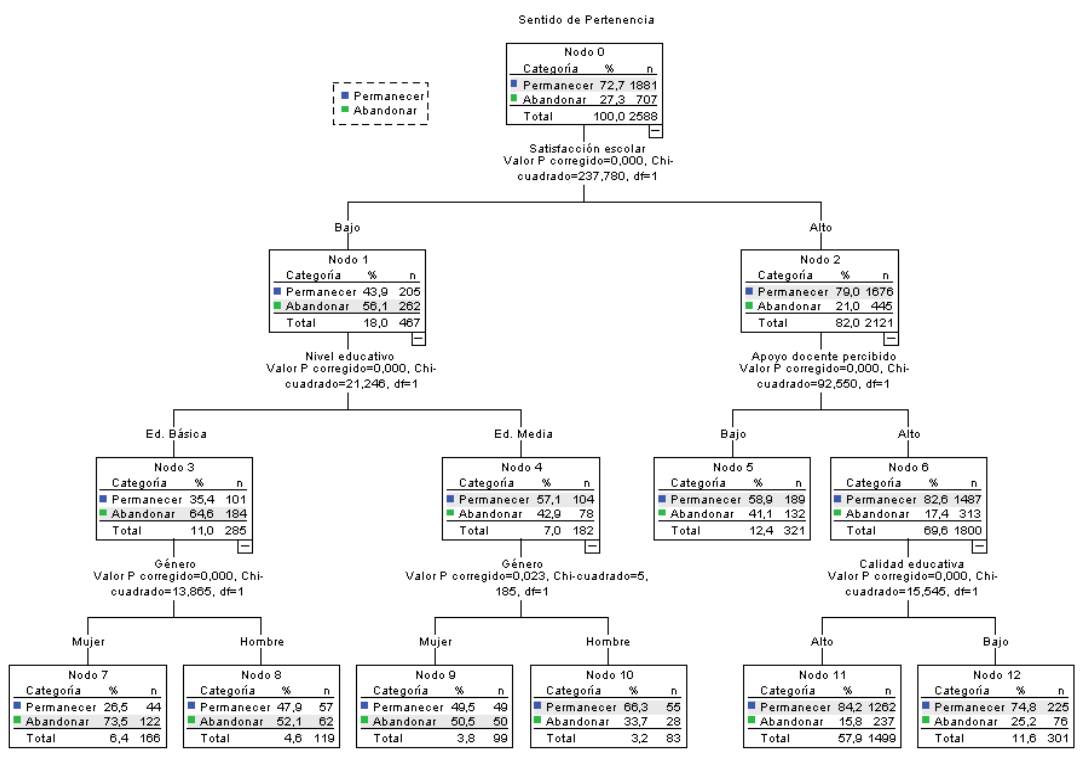

Gráfica 1. Sentido de pertenencia a la escuela en NNA

Fuente: elaboración propia con base los resultados de la EDH-NNA (2017).

Aunque el bienestar escolar es influenciado por factores ajenos a la escuela, como el apoyo parental o la autopercepción de competencias académicas, también es resultado de la evaluación que se hace de la experiencia social desarrollada en el espacio educativo (OCDE, 2017). En su comunidad escolar el estudiante debería obtener una satisfacción emocional 
y afectiva como resultado de un conjunto de experiencias gratificantes que refuerzan el sentido de pertenencia e identidad, las cuales contribuyan a la definición de proyectos de vida y construcción de espacios donde pueda sentirse emocionalmente a salvo (Blanco, R. 2008; Hopenhayn y Sojo, 2011), aun cuando esta valoración no esté acompañada por el logro académico.

Por ser la escuela un espacio de intersubjetividad con mutuas afectaciones (Martínez, 2007), es allí donde los actores construyen redes activas de vínculos sociales y afectivos que, según su extensión y fortaleza, inciden en el nivel de apego escolar. En la medida en que la experiencia es gratificante y los lazos establecidos con compañeros y docentes son sólidos y de calidad, el estudiante se siente integrado e indispensable para el grupo, lo que mejora su grado de implicación y sentimiento de seguridad. Resultados de investigaciones de este tipo han sido presentados por Garbanzo (2007), Mesurado (2010), Osorio y Pérez (2010), Casas et al. (2014) y Alfaro et al. (2016), quienes destacan que altos niveles de satisfacción escolar están asociados a una mejor predisposición y afrontamiento de desafíos educativos, motivación y logro exhibido, además de una reducción en las tasas de abandono y fracaso escolar.

En un segundo nivel jerárquico de segmentación encontramos las variables Nivel educativo y Apoyo docente percibido, según se manifieste una baja o alta satisfacción con la experiencia escolar respectivamente. En el segmento de baja Satisfacción escolar [nodo 1], el bajo Sentido de pertenencia incrementa entre quienes cursan Educación Básica [ $\left.x^{2}=21,246 ; p=0,000\right]$. Aproximadamente 2 de cada 3 estudiantes en este ciclo (64,6 \%) estarían dispuestos a migrar de su establecimiento esducativo, cifra significativamente mayor a la de sus pares en Educación Media (42,9 \%).

Al respecto, como tanto la infancia como la preadolescencia son periodos marcados por la maduración física, cognitiva y psicológica, además de la exploración y selección de categorías identitarias (Massenzana, 2017), los estudiantes de enseñanza básica son más sensibles a los conflictos que pueden ocurrir entre pares y el centro educativo. A su vez, la transición hacia el ciclo secundario puede estimular el deseo de migrar del establecimiento, por cuanto las elecciones parentales educativas tienden a definirse al inicio y fin de la Enseñanza Básica [nodo 3]. Por su parte, los estudiantes de Enseñanza Media serían menos sensibles a las eventualidades del medio escolar dada la etapa de desarrollo biopsicosocial en que se encuentran y la pronta finalización de su trayectoria escolar. Estos factores presionarían hacia la conformidad en lugar de tener que reiniciar procesos de adaptación académica y relación con otros.

En el itinerario opuesto, o perfil protector (alto nivel de Satisfacción escolar; nodo 2], el grupo se segmenta con base en el Apoyo docente percibido $\left[x^{2}=92,550 ; p=0,000\right]$. A medida que el nivel de implicancia, preocupación y acompañamiento percibido por parte de los docentes incrementa, 
lo hace también el Sentido de pertenencia (82,9\%). Para los estudiantes el docente es un otro significativo en términos de soporte académico y emocional; la fortaleza de este vínculo afecta positivamente el apego estudiantil con su comunidad educativa. Cuando el profesor sostiene una relación cercana posibilita que el estudiante desarrolle una actitud positiva y se sienta seguro al encontrarse en un espacio de cuidado y protección adicional al que le brinda su familia (Juvonen, 2006; Luckner y Pianta, 2011).

En general, como varios investigadores informan, la calidad del vínculo entre docente y estudiante es un recurso simbólico de importancia para la optimización de las relaciones y condiciones de aula que revitalizan su preocupación por el aprendizaje y satisfacción con los componentes disciplinares, instruccionales y pedagógicos (Albornoz y Cornejo, 2017). De este modo, en la medida en que los alumnos hacen una buena evaluación de su profesor, por cuanto declaran recibir respeto y amabilidad (99,3 \%), motivación a seguir aprendiendo (98,8\%), estímulos a sus capacidades (97,8\%), espacio para expresar opiniones $(98,2 \%)$ y consideración de sus aportes y sugerencias (95,5\%), se acrecienta el Sentido de pertenencia [nodo 6].

En un tercer nivel jerárquico (dentro de la tipificación del perfil de riesgo) aparece el Género como atributo significativo $\left[x^{2}=13,865 ; p=0,000\right]$. Entre quienes tienen una baja Satisfacción escolar y están cursando Educación Básica, el hecho de ser mujer acentúa el deseo de migrar (73,5\%), aunque la situación para los hombres es igualmente alta y preocupante (52,1\%).

Por su parte, en el perfil protector la Calidad educativa se posiciona como último rasgo distintivo de segmentación $\left[x^{2}=15,545 ; p=0,000\right]$. Los estudiantes que tienen una alta valoración de las experiencias de aprendizaje y oportunidades que la escuela otorga para su desarrollo integral manifiestan mayoritariamente un deseo por permanecer o de alto Sentido de pertenencia (84,2\%). En efecto, el alumnado que pertenece a este grupo es quien expresa una mayor conformidad en términos de Acuerdo y Muy de acuerdo respecto a que su colegio entrega buena educación $(99,4 \%)$, da la posibilidad de elegir actividades de interés (95,9\%), tiene los recursos necesarios para enseñar $(98,6 \%$ e e incentiva la valoración de la diversidad (98,7 \%) y la participación y organización estudiantil $(98,1 \%$ ).

En consecuencia, mientras en el perfil de riesgo un 73,5\% del alumnado manifiesta desear migrar de su unidad educativa, un $84,2 \%$ del perfil protector expresa permanecer, lo que en términos porcentuales representa un aumento del $169 \%$ y un 15,8 \% respectivamente [con relación al nodo 0]. El sentido de pertenencia con la escuela se configura como un fenómeno multidimensional afectado por características relacionales y emocionales que, al ser la escuela un espacio de interacción, interpretación y filiación con otros, son fortalecidas o mitigadas según la calidad de los vínculos, particularmente con los docentes y la institución. 
Tabla 3.

Perfil de mayor riesgo a la migración escolar $(\%)(n=122)^{*}$

\begin{tabular}{lccc}
\hline & General & Perfil & Diferencia de tasas \\
\hline AFP bajo & 17,8 & 38,5 & 116,3 \\
\hline ADP bajo & 18,5 & 41,0 & 121,6 \\
\hline Sat. amigos baja & 17,3 & 40,2 & 132,4 \\
\hline Sat. con la vida baja & 18,1 & 50,8 & 180,7 \\
\hline Autoestima baja & 40,1 & 61,5 & 53,4 \\
\hline Dependencia & & & $-23,3$ \\
- Municipal & 43,8 & 33,6 & 15,9 \\
- Part. subvencionado & 50,9 & 59,0 & 42,3 \\
- Part. pagado & 5,2 & 7,4 & \\
\hline
\end{tabular}

* Definido con base en Género (mujeres), Nivel educativo (Básico) y Nivel de satisfacción escolar (bajo); AFP = Apoyo familiar Percibido; ADP = Apoyo docente percibido; Sat. = Satisfacción; Part. $=$ Particular

Fuente: elaboración propia con base en los resultados de la EDH-NNA (2O17).

De acuerdo con el modelamiento por árboles de segmentación, serían las mujeres que cursan Enseñanza Básica y manifiestan baja satisfacción con su experiencia educativa quienes constituyen el perfil de mayor riesgo a la migración escolar. Este grupo dista significativamente de la tendencia general en cuatro atributos clave: perciben un bajo apoyo familiar (+116\%) y docente $(+121 \%)$, tienen una baja autoestima $(+53 \%)$ y satisfacción con la vida (+180\%); además, en su mayoría pertenecen a establecimientos particulares subvencionados (59,0\%).

Tabla 4.

Perfil con mayor sentido de pertenencia a la escuela (\%) ( $n=1262)^{*}$

\begin{tabular}{lccc}
\hline & General & Perfil & Diferencia de tasas \\
\hline Autoestima alta & 59,9 & 71,9 & 20,0 \\
\hline AFp alto & 82,2 & 89,5 & 8,9 \\
\hline Sat. con la vida alta & 81,9 & 89,2 & 8,9 \\
\hline Dependencia & & & 0,7 \\
- Municipal & 43,8 & 44,1 & $-2,2$ \\
- Part. sub. & 50,9 & 49,8 & 17,3 \\
- Part. pagado & 5,2 & 6,1 & \\
\hline
\end{tabular}

* definido con base a Satisfacción escolar (alta), Apoyo docente percibido (alto) y Percepción de calidad educativa (alta); AfP = Apoyo familiar percibido; Sat. = Satisfacción; Part. = Particular. Fuente: elaboración propia con base en los resultados de la EDH-NNA (2017). 
Los estudiantes que expresan una alta valoración en cuanto a Satisfacción escolar, Apoyo docente percibido y Calidad educativa representan el perfil con mayor Sentido de pertenencia y constituyen el 48,7 \% del total de la muestra. Dentro de los atributos distintivos de este conglomerado respecto del estudiante general, encontramos: Autoestima alta $(+20,0 \%)$; Apoyo familiar percibido (+8,9\%); Alta satisfacción con la vida (+8,9\%) y pertenecer a una dependencia Particular pagada (+17,3\%).

La movilidad escolar puede ser positiva si se trata de desplazamiento estudiantil de una escuela a otra en busca de una mayor calidad educativa, situación que tributaría en los procesos de aprendizaje del alumno que migra y en el mejoramiento del sistema educativo en su conjunto (Eyzaguirre y Fontaine, 2008). De hecho, la lógica voucher y subvención escolar se inspira en el principio de la libre elección parental, asumiendo a la familia como un actor clave que puede tomar decisiones libres, racionales e informadas en la búsqueda del establecimiento que mejor satisfaga sus expectativas. No obstante, este argumento presenta serias debilidades y contradicciones para su materialización en el contexto educativo de acuerdo con la evidencia disponible.

\section{Conclusiones}

Junto con la familia, a la escuela le asiste la responsabilidad de constituirse como un lugar de protección y seguridad, encargado de proveer las herramientas y competencias necesarias para el desarrollo integral. En la medida en que se establecen relaciones sólidas y de calidad con profesores y compañeros, la experiencia escolar es gratificante, mejora la disposición a los desafíos académicos y se fortalece el nivel de implicación y apego hacia la institución. No obstante, no todos los estudiantes logran forjar un vínculo afectivo estable y duradero con ella.

Atendiendo a la relevancia que tiene el sentido de pertenencia sobre el rendimiento académico, el sentimiento de bienestar y la construcción de comunidad, este artículo perfila, mediante la técnica multivariante de árboles de segmentación, los itinerarios de protección y riesgo frente al deseo de permanecer o abandonar la unidad educativa. A nivel general, las variables de mayor capacidad discriminativa y en orden jerárquico fueron Satisfacción escolar, Nivel educativo, Apoyo docente percibido, Género y Calidad educativa.

El sentido de pertenencia con la escuela aumenta a medida que mejoran el nivel de Satisfacción escolar, el Apoyo docente percibido y la evaluación de Calidad educativa. Estos factores estarían en directa relación con las posibilidades de llevar a cabo proyectos personales y colectivos tendientes al afianzamiento de las relaciones y la identidad. 
Como contraparte, el desapego respecto de la institución se intensifica en contextos escolares escasamente gratificantes, en particular para las mujeres que cursan el nivel básico de enseñanza. La alta prevalencia del deseo de migrar sería expresión de la debilidad evidenciada por la escuela para constituirse en una instancia de desarrollo del estudiante que, junto con desafiarlo intelectualmente, sea capaz de brindarle el espacio de seguridad, protección y confort que precisa.

Al proveer experiencias educativas emocionalmente gratificantes e intelectualmente desafiantes, con profesores que los motiven, respeten y estimen, el estudiantado se sentirá más acogido, más seguro respecto de sus capacidades y desarrollará un mayor vínculo con su institución educativa. En experiencias investigativas similares esta implicación ha demostrado relación con el mejoramiento de las condiciones de educabilidad para el aprendizaje escolar, el bienestar subjetivo y sentido de comunidad; estos factores son pilar de la nueva mirada amplia de calidad que las políticas post-reforma han tratado de instaurar los últimos años, y resultados como los aquí expuestos contribuyen directamente a la focalización de esfuerzos y recursos con el objeto de integrar en las mediciones de calidad el aspecto relacional y social de la escuela.

En conclusión, el nivel de implicación y apego para con la institución educativa se construye sobre la base de un conjunto de indicadores perceptivos de naturaleza individual que, pudiendo o no estar en sintonía con la evidencia objetiva que la sustenta, estructura un sentimiento de filiación, un modo de sentirse integrado, único e irremplazable para el grupo en particular y dentro de la comunidad educativa en general. Los beneficios de este hecho son múltiples, pero se destacan los vinculados a la satisfacción escolar y el apoyo docente y calidad educativa percibidos en la escuela a la que se pertenece.

\section{Referencias}

Agencia de Calidad de la Educación. (19 de abril de 2017). PISA: Bienestar escolar impacta fuertemente en la satisfacción de los estudiantes chilenos con su vida. https://www.agenciaeducacion.cl/noticias/ pisa-bienestar-escolar-impacta-fuertemente-la-satisfaccion-los-estudiantes-chilenos-vida/

Albornoz, N. y Cornejo, R. (2017). Discursos docentes sobre el vínculo con sus estudiantes: tensiones, enfrentamiento y distancia. Estudios Pedagógicos, 43(2), 7-25.

Alfaro, J., Guzmán, J., Reyes, F., García, C., Varela, J. y Sirlopú, D. (2016). Satisfacción global con la vida y satisfacción escolar en estudiantes chilenos. Psykhe, 25(2), 1-14. 
Aron, A. M., Milicic, N. y Armijo, I. (2012). Clima social escolar: una escala de evaluación-Escala de Clima Social Escolar, Eclis. Universitas Psychologica, 11(3), 803-813.

Assaél, J. (2011). La empresa educativa chilena. Educação e Sociedade, 32(115), 305-322.

Bailey, T., Smith, S. y Jenkins, D. (2015). Redesigning America's community colleges. Harvard University Press.

Bellei, C. (2013). El estudio de la segregación socioeconómica y académica de la educación chilena. Estudios Pedagógicos, 39, 325-345.

Bellei, C. (2015). El gran experimento: Mercado y privatización de la educación chilena. LOM.

Blanco, J. (2010). Violencia y delincuencia juvenil: desafíos en relación con los programas y respuestas del sistema. Conceptos, 18, 1-13.

Blanco, R. (2008). Construyendo las bases de la inclusión y la calidad de la educación en la primera infancia. Revista de Educación, 347, 33-54.

Carbajal, P. (2010). La convivencia como alternativa a la violencia escolar: experiencias exitosas desde Latinoamérica. [Conferencia] VII Encuentro Participación de la Mujer en la Ciencia. Centro de Investigaciones en Óptica (memoria en disco compacto).

Carrasco, A. y González, P. (2017). Liderazgo para la inclusión y para la justicia social: el desafío del liderazgo directivo ante la implementación de la Ley de inclusión escolar de Chile. Educación y Ciudad, 33, 63-74.

Carrasco, C. y Luzón, A. (2019). Respeto docente y convivencia escolar: significados y estrategias en escuelas chilenas. Psicoperspectivas, 18(1), 64-74.

Casas, F., Sarriera, J., Alfaro, J., González, M., Figuer, C., Abs da Cruz, D., Bedín, L., Valdenegro, B. y Oyarzún, D. (2014). Satisfacción escolar y bienestar subjetivo en la adolescencia: poniendo a prueba indicadores para su medición comparativa en Brasil, Chile y España. Suma Psicológica, 21, 70-80.

Cordano, M. (8 de mayo del 2017). El sentido de pertenencia al colegio es un factor clave en el bienestar de los alumnos. http://www.economiaynegocios.cl/noticias/noticias.asp?id = 358589

Espinoza, O. (2015). Equidad en inclusión en el sistema de educación superior de Chile. En O. Espinoza, Equidad e inclusividad en la educación superior en los países andinos: los casos de Bolivia, Chile, Colombia y Perú (vol. 38, pp. 93-132). Ediciones Universidad Ucinf.

Eyzaguirre, B. y Fontaine, L. (2008). Las escuelas que tenemos. Centro de Estudios Públicos.

Fernández, M., Mena, L. y Riviere, J. (2010). Fracaso y abandono escolar en España. Obra Social Fundación la Caixa. Estudios Sociales n. ${ }^{\circ} 29$. 
Fierro, M. (2013). Convivencia inclusiva y democrática: una perspectiva para gestionar la seguridad escolar. Sinéctica, 40, 1-18.

Finn, J. (1989). Withdrawing from school. Review of Educational Research, 59(2), 117-142.

Garbanzo, G. (2007). Factores asociados al rendimiento académico en estudiantes universitarios, una reflexión desde la calidad de la educación superior pública. Revista Educación, 31(1), 43-63.

Gervilla, E. y Palmer, A. (2009). Predicción del consumo de cocaína en adolescentes mediante árboles de decisión. Revista de Investigación en Educación, 6, 7-13.

Gilbert, J. (2010). Introducción a la sociología. LOM.

Han, J. y Kamber, M. (2006). Data mining: Concepts and techniques. Morgan Kaufmann.

Hopenhayn, M. y Sojo, A. (eds.). (2011). Sentido de pertenencia en sociedades fragmentadas: América Latina desde una perspectiva grobal. Siglo xxI.

John, E., Daun, N. y Moronski, K. (2018). Public policy and higher education: Reframing strategies for preparation, access, and college success. Routledge.

Johnson, B. (2010). Exploring and explicating the distinctive features of educational organizations: Theories and theorizing. En W. Hoy y M. DiPaola's (eds.), Analyzing school context: Influences of principals and teachers in the service of students (pp. 1-38). IAP.

Juvonen, J. (2006). Sense of belonging, social bonds, and school functioning. En P. Alexander y P. Winne (eds.), Handbook of Educational Psychology. Routledge.

Keating, A. y Janmaat, G. (2016). Education through citizenship at school: Do school activities have a lasting impact on youth political engagement? Parliamentary Affairs, 69, 409-429.

Keating, A., Kerr, D., Lopes, J., Featherstone, G. y Benton, T. (2009). Embedding Citizenship Education (CE) in Secondary Schools in England (2002-08): Citizenship Education Longitudinal Study (CELS). Seventh Annual Report. DCSF.

Kia-Keating, M. y Ellis, B. (2007). Belonging and connection to school in resettlement: Young Refugees, School belonging, and psychosocial adjustment. Clinical Child Psychology and Psychiartry, 12(1), 29-43.

López, V., Bilbao, M., Ascorra, P., Moya, I. y Morales, M. (2014). La Escala de clima escolar: Adaptación al español y validación en estudiantes chilenos. Universitas Psychologica, 13(3), 1111-1122.

López de Mesa, C., Carvajal, C., Soto, M. F. y Urrea, P. (2013). Factores asociados a la convivencia escolar en adolescentes. Educación y Educadores, 16(3), 383-410. 
Luckner, A. y Pianta, R. (2011). Teacher-student interaction in fifth grade classrooms: Relation with children's peer behavior. Journal of Applied Developmental Psychology, 32(5), 257-266.

Martínez, D. (2007). Nuevas regulaciones, ¿nuevos sujetos? En M. Feldfeber y D. Oliveira, Políticas educativas y trabajo docente: nuevas regulaciones, ¿nuevos sujetos? Noveduc.

Massenzana, F. (2017). Autoconcepto y autoestima: ¿sinónimos o constructos complementarios? \{Psocial\}, 3(1), 39-52.

Mesurado, B. (2010). La experiencia de Flow o Experiencia Óptima en el ámbito educativo. Revista Latinoamericana de Psicología, 42(2), 183-192.

Murillo, F. y Duk, C. (2016). Segregación escolar e inclusión. Revista Latinoamericana de Educación Inclusiva, 10(2), 11-13.

Orellana, E. y Segovia, J. (2014). Evaluación del clima social escolar mediante semilleros de convivencia de los octavos de educación general básica (tesis de pregrado). Universidad de Cuenca, Cuenca, Ecuador. http://dspace.ucuenca.edu.ec/jspui/bitstream/123456789/5022/1/Tesis.pdf

Organización para la Cooperación y el Desarrollo Económicos-OCDE. (2017). ¿Son felices los alumnos? Resultados de PISA 2015: el bienestar de los estudiantes.

Osorio, J. y Pérez, K. (2010). El nivel de satisfacción escolar y su relación con la orientación vocacional en alumnos de psicología educativa (tesis profesional). Universidad Pedagógica Nacional de México.

Peña, C. (2007). Por qué no debemos seleccionar. En J. J. Brunner y C. Peña (eds.), La reforma al sistema escolar: aportes para el debate (pp. 245-252). Universidad Diego Portales.

PISA. (2015). Informe de resultados PISA 2015 Competencia científica, lectora y matemática en estudiantes de quince años en Chile. Agencia de Calidad de la Educación.

Quaresma, M. (2014). Entre o herdado, o vivido e o projetado. Estudo de caso sobre o sucesso educativo em dois colégios privados frequentados pelas classes dominant. Edições Afrontamento.

Quaresma, M. y Zamorano, L. (2016). El sentido de pertenencia en escuelas públicas de excelencia. Revista Mexicana de Investigación Educativa, 21(68), 275-298.

Riley, K. (2013). Leadership of place. Stories froms schools in the US, UK and South Africa. Bloomsbury.

Román, M. y Murillo, J. (2011). América Latina: violencia entre estudiantes y desempeño escolar. Revista Cepal, 104. 
Sánchez, B., Colón, Y. y Esparza, P. (2005). The role of sense of school belonging and gender in the academic adjustment of Latino adolescents. Journal of Youth and Adolescence, 34(6), 619-628.

Spady, W. (1971). Dropouts from Higher Education: Toward an empirical model. Interchange, 2, 38-62.

Tedesco, J. C. (2012). Educación y justicia social en América Latina. Fondo de Cultura Económica: Unsam.

Tijmes, C. (2012). Violencia y clima escolar en establecimientos educacionales en contextos de alta vulnerabilidad social de Santiago de Chile. Psyhke, 21(2), 105-117.

Tinto, V. (1975). Dropout education: A theoretical synthesis of recent research. Review of Educational Research, 45, 89-125.

Valenzuela, J. (2007). Más allá de la tarea: pistas para una redifinición del concepto de motivación escolar. Educacao e pesquisa, 33(3), 409-426.

Valenzuela, J. P., Bellei, C. y De los Ríos, D. (2010). Segregación escolar en Chile. En S. Martinic y G. Elacqua (eds.), Cambios en la gobernanza del sistema educativo chileno (pp. 257-284). Unesco, Pontificia Universidad Católica de Chile.

Villalobos, B., Carrasco, C., Olavarría, D., Ortiz, S., López, V., Oyarzún, D., Ascorra, P. Ayala, A., Bilbao, A., Morales, M. y Álvarez, J. P. (2016). Victimización de pares y satisfacción con la vida: la influencia del apoyo de profesores y compañeros de la escuela. Psykhe, 25(2), 1-16. 